\title{
Estimating GFR and GFR decline in patients with T2DM
}

The use of serum creatinine-based formulas to identify patients with type 2 diabetes mellitus (T2DM) who are at increased risk of accelerated renal function decline and to monitor renal disease progression in this population should be questioned, say researchers. Flavio Gaspari and colleagues found that in patients with T2DM and either normoalbuminuria or microalbuminuria, estimation formulas failed to detect glomerular hyperfiltration and did not reliably describe changes in glomerular filtration rate (GFR) over time.

Although different methods can be used to accurately measure GFR, they can be difficult to apply, especially in routine clinical practice. Formulas based on serum creatinine level have therefore been used to estimate GFR and avoid the need for direct GFR measurements. "The first estimation formula was proposed in 1957 , and since then, more than 20 different formulas have been introduced," says researcher Esteban Porrini. The accuracy of the most widely used formulas has been questioned, however, particularly in patients with normal or increased GFR. In addition, the performance of the most recent equation, the CKD-EPI equation, has been questioned in patients with diabetes. "This is a major issue as reliable estimation of renal function trajectory is instrumental to monitor renal disease progression and the effect of treatment..." say the researchers.

To assess the accuracy of GFR estimation formulas, Gaspari et al. compared 15 formulas against measured GFR values in patients with T2DM from the BENEDICT-B and DEMAND trials. Baseline GFR had been measured in 600 participants and 6-monthly serial measurements of iohexol plasma clearance over a median period of 4 years had been performed in 449 individuals.

The researchers found that the overall agreement between measured and estimated GFR was poor for all formulas. In hyperfiltrating patients, the estimation formulas underestimated GFR and failed to detect a 6-month reduction in measured GFR. 58 of 90 hyperfiltrating patients were not identified as having hyperfiltration by any formula. The formulas also underestimated long-term GFR decline across the whole study group, as well as in hyperfiltering, normofiltering and hypofiltering subgroup analyses. Five formulas generated false positive slopes

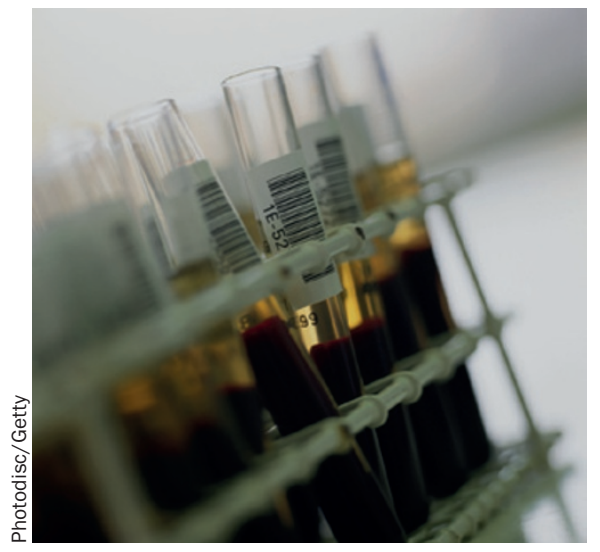

in hyperfiltering patients. "We confirmed that none of the 15 most commonly used formulas reliably estimates actual GFR in patients with T2DM. Moreover, we found that none of them accurately describes GFR changes over time," states Porrini.

The researchers say that future research should focus on designing new formulas to more accurately describe kidney function in patients with diabetes and, conceivably, to guide drug dosing in this population. "In the meantime, serial GFR measurements are instrumental to evaluate renal function trajectory in this population," concludes Gaspari.

Susan J. Allison

Original article Gaspari, F. et al. The GFR and GFR decline cannot be accurately estimated in type 2 diabetics. Kidney Int. doi:10.1038/ki.2013.47 